\title{
Tetrahedral-Mesh Simulations of Shock-Turbulence Interaction
}

\author{
Balaji Shankar Venkatachari* \\ National Institute of Aerospace, Hampton, VA 23666 \\ and \\ Chau-Lyan Chang ${ }^{\dagger}$ \\ NASA Langley Research Center, Hampton, VA 23681
}

Despite decades of development of unstructured mesh methods, direct numerical simulations (DNS) of turbulent flows are still predominantly performed on structured or unstructured hexahedral meshes with high-order finite-difference methods, weighted essentially nonoscillatory (WENO) schemes, or hybrid schemes formed by their combinations. Tetrahedral meshes offer easy mesh generation and adaptation around complex geometries and the potential of an orientation-free grid that would benefit the isotropic nature of small-scale dissipation, as well as the solution accuracy of intermediate scales. To advance the state of the art of unstructured-mesh simulation capabilities for shock/turbulence interaction, DNS using pure tetrahedral meshes are carried out with the space-time conservation element, solution element (CESE) method in this research. By its design, the CESE method is constructed based on a non-dissipative scheme and is a genuinely multidimensional numerical framework that is free from the use of an approximate Riemann-solver. The numerical framework also provides the ability to add numerical dissipation (the nondissipative scheme acts as the reference state like that of the reversible state in thermodynamics) when needed (with justification from mathematics/physics). The above-mentioned features along with the CESE method's consistent shock-capturing approach and strong enforcement of flux conservation in spacetime offers a novel method to accurately simulate turbulent flows and their interaction with shocks using tetrahedral meshes. Two canonical problems, namely, isotropic turbulence interaction with a normal shock and a Mach 2.9 turbulent boundary layer flow over a $24^{\circ}$ compression corner are investigated in this study. Computational results show reasonably good agreement with experimental data and results from structured-mesh, high-order simulations available in the literature. Successful validation of these canonical problems demonstrated here paves the way for future high-fidelity supersonic flow simulations involving complex-geometries.

\section{Nomenclature}

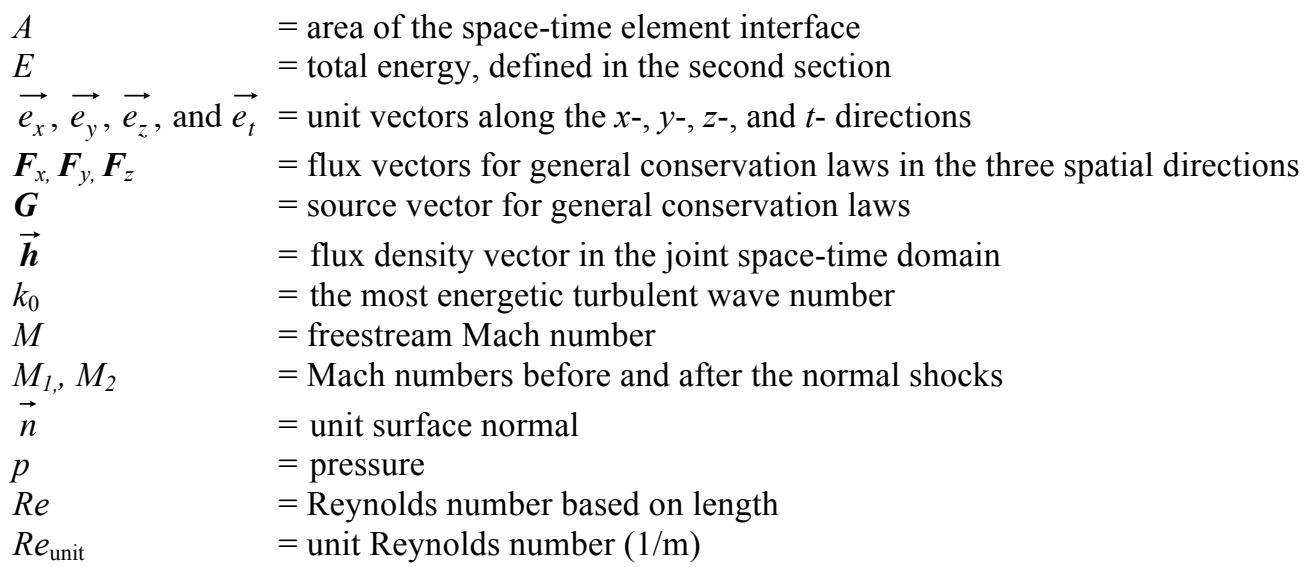

\footnotetext{
*Research Engineer, Email: balaji.s.venkatachari@nasa.gov, Senior member, AIAA

${ }^{\dagger}$ Aerospace Technologist, Computational AeroSciences Branch, Email: Chau-Lyan.Chang@nasa.gov, Associate Fellow, AIAA
} 


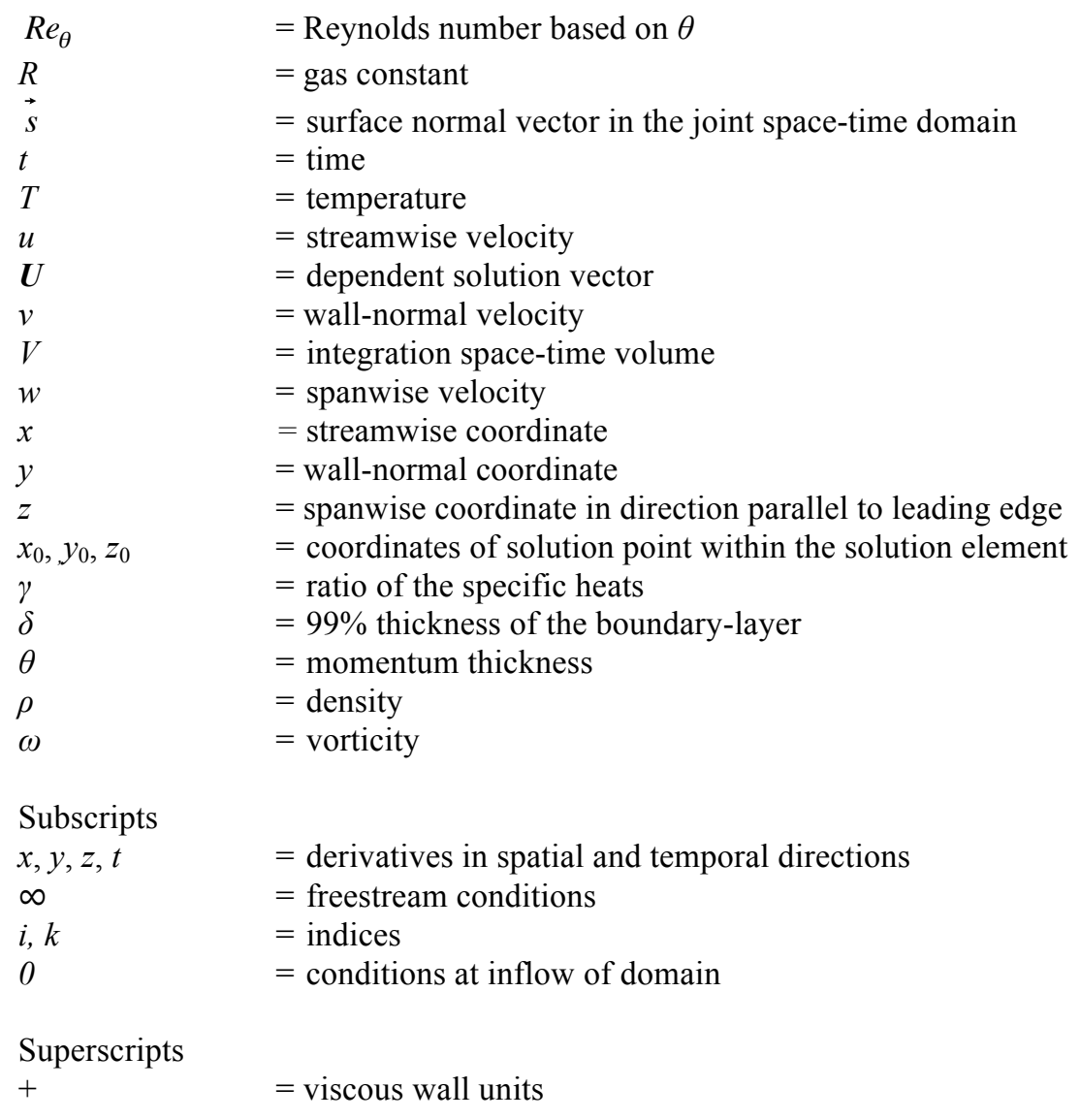

\section{Introduction}

Numerical simulations of turbulent flows have been actively pursued for several decades. Parallel to the advancement of CPU speed and large-scale parallel clusters, mesh size for turbulent flow simulations is approaching trillions of grid points. ${ }^{1}$ The state-of-the-art large-scale direct numerical simulation (DNS) done in Ref. 1 simulated isotropic turbulence interacting with shocks using about 2 million CPU cores. Three main ingredients went into the one of a kind simulation in Ref. 1: (i) structured meshes; (ii) a hybrid numerical scheme that uses $6^{\text {th }}$-order central-difference scheme in smooth regions of the flow and a $5^{\text {th }}$ order weighted essentially nonoscillatory (WENO) scheme for shock-capturing; and (iii) an explicit timemarching scheme. Many other similar investigations also share these common characteristics.

Despite decades of development in methods for generating unstructured meshes, as well as numerical methods that use them, DNS or large eddy simulations (LES) of turbulent flows have consistently relied upon the use of structured meshes. The primary reason is that the higher-order numerical methods employed in these simulations are based on finite-difference methods. Even when unstructuredmesh-based higher-order schemes are employed for DNS/LES, there is a preference toward using hexahedral elements because of the difficulties the numerical methods have in handling tetrahedral elements especially in regions of shocks or sharp gradients. ${ }^{2,3}$ From the standpoint of turbulent flow physics, unstructured meshes with isotropic tetrahedral meshes are in general free of any artificial grid orientation and therefore, are closer to the isotropic nature of the smaller dissipative scales in those flows. Hence, one could argue that such isotropic meshes in conjunction with proper (nonexcessive) numerical dissipation could improve the solution accuracy of medium-to-small scales in DNS or LES. However, the above-mentioned reasoning remains to be proven. For turbulent boundary-layer simulations, the use of pure isotropic tetrahedral meshes near the wall would also significantly increase the grid count by orders of magnitude, making it intangible for most of the high-performance computing facilities. 
Implicit schemes have been preferred for many CFD computations due to their robustness to deal with stiffness (either from embedded poor-quality mesh elements or stiff source terms) and rapid convergence to steady state solutions. However, when applied to large-scale DNS/LES of turbulent flow simulations (and transitional flow or acoustic-wave computations), the ability to march with a larger time step is lost as the solution accuracy dictates the time step instead of the convergence rate to steady state. From a flow physics standpoint, the physical time scale in most unsteady, transitional, or turbulent flows is quite close to that of the acoustic wave. This implies that the time step determined from the CFL number of about 1 is very close to the physical time scale. Furthermore, as the mesh size increases to the order of billions or trillions, the accompanying matrices in implicit schemes brings in additional complexities: (i) the increased matrix size makes the memory usage suboptimal under the large-scale cluster environment; and (ii) parallel scaling for banded matrices have also been poor for the current computer architecture. Thereby, explicit schemes free of matrix solvers with high-order time accuracy become more efficient than implicit schemes for such flow simulations.

Shock/turbulence interaction is fundamental to many fluid mechanics applications, especially in the area of high-speed flights where shock wave - turbulent boundary layer interaction (SWBLI) is a dominant feature. As a result, various aspects of this problem have been the subject of many DNS/LES studies (e.g., see Refs. 4-7). All of these studies employ either compact-differencing schemes with highorder filters, ${ }^{8}$ WENO methods, ${ }^{9}$ or a hybrid treatment ${ }^{1}$ that uses the upwind-based WENO operator to stabilize flow discontinuities and switches to low-dissipation schemes away from the shock to resolve the unsteady waves. However, a previous study ${ }^{10}$ has shown that any use of an upwind-based approach to capture shock effects, when not kept to a minimum, can significantly affect the post-shock turbulence. Furthermore, all of the above-mentioned numerical schemes can only be implemented on structured meshes.

In light of the discussion above, this paper investigates numerical simulations of turbulent flows and their interaction with shocks by using tetrahedral meshes along with a unified numerical framework that is time-explicit and has a strong conservation preserving property. Others are pursuing similar research in the CFD community. For example, Khalighi et al. ${ }^{11}$ investigated explicit, hybrid algorithms for unstructured meshes and validated their algorithms and codes for turbulent jets and the accompanying acoustic wave propagation. In the present research, the space-time conservation element, solution element (CESE) method ${ }^{12-17}$ is used for turbulent flow simulations with tetrahedral meshes. The CESE method is formulated with the strong space-time unity integral form of conservation laws making it suitable for handling waves and flow discontinuities with high accuracy. ${ }^{17-19}$ The concept of flux conservation in time that is introduced in the CESE method removes any distinction between space and time, thereby providing uniform accuracy in space and time and that feature has appreciable advantages for eddy-resolving turbulent flow computations. Accuracy of a temporal scheme is critical for the simulation of turbulent flows and inadequate temporal discretization can significantly lower the accuracy of spatial schemes. ${ }^{20}$ Additionally, the time-accurate local time-stepping (TALTS) scheme ${ }^{15-17}$ formulated in the CESE method is appealing for eddy-resolving turbulent simulations due to a wide spectrum of length and time scales involved in such flows. The TALTS algorithm allows for larger time steps to be used for large flow structures away from the wall and in the quiet freestream regions, but at the same time guaranteeing conservation of fluxes in space and time. The genuinely multidimensional formulation, free of approximate Riemann solvers and dimensional splitting, of the CESE method offers a distinctly different way to simulate turbulent flows interacting with shocks. Whether or not this distinctness translates into significant advantages is also explored through this work.

The main objective of this paper is to advance the state-of-the-art direct numerical simulations for turbulent flows with pure tetrahedral meshes. Successful demonstration of such simulations will constitute the building blocks for future high-fidelity computations involving complex geometries. Towards this end, two canonical problems are investigated as part of the study: (i) isotropic turbulence/normal shock interaction; and (ii) interaction of a supersonic turbulent boundary layer with an oblique shock.

The first problem studied in this work is a simplistic representation of the problem of shockturbulence interaction and is a continuation of the authors' previous work. ${ }^{21}$ The problem also serves as a stringent test for assessing the capability of a numerical method to handle the passing of smaller flow structures through a strong shock. The second problem investigated here is the interaction of a Mach 2.9 turbulent boundary layer with an oblique shock originating from a $24^{\circ}$ compression corner. The topic of SWBLI has been an active research area both computationally and experimentally for many decades. The flow physics, numerical issues and the state of the art for its prediction have been summarized in several 
reviews. ${ }^{22-25}$ Despite so many studies, some aspects of the flow physics are still not well understood. Moreover, almost all of the experimental studies have been performed at relatively high Reynolds numbers that are beyond current DNS/LES capabilities. To rectify that aspect, Bookey et al., ${ }^{26}$ and Ringuette et al. ${ }^{27}$ performed experiments of a Mach 2.9 supersonic turbulent boundary layer flow past a $24^{\circ}$ compression corner under flow conditions that were within DNS accessible range (Reynolds number, $\operatorname{Re}_{\theta} \approx 2400$ ). The same configuration is investigated here using the CESE method and tetrahedral meshes. This canonical case has also been studied in other DNS investigations ${ }^{7,28}$ using hexahedral meshes. The results from CESE are compared in detail with experimental measurements ${ }^{27}$ as well as data from the DNS studies. ${ }^{7,28}$

A brief description of the numerical method and the code used is given in the next two sections, followed by the results section in which computational results of the above-mentioned cases are given in sequence. Flow physics due to the interaction with shocks is discussed in detail to demonstrate that the current unstructured mesh DNS is a viable alternative to more conventional structured mesh based methods in high-fidelity simulations of supersonic turbulent flows and their interaction with shocks.

\section{The CESE Method}

Consider any 3D conservation law (Navier-Stokes, Maxwell's equations etc.). Let: (a) $x, y$, and $z$

be the spatial coordinates, and $t$ be the time coordinate; (b) $x_{1} \stackrel{\text { def }}{=} x, x_{2} \stackrel{\text { def }}{=} y, x_{3} \stackrel{\text { def }}{=} z$, and $x_{4} \stackrel{\text { def }}{=} t$ be the coordinates of a four-dimensional Euclidean space $E_{4}$; (c) $\overrightarrow{e_{x}}, \overrightarrow{e_{y}}, \overrightarrow{e_{z}}$, and $\overrightarrow{e_{t}}$ be the unit vectors along the $x$-, $y$-, $z$-, and $t$-directions, respectively; and (d) $\overrightarrow{\boldsymbol{h}}$, be the space-time flux density vector, respectively. Then $\overrightarrow{\boldsymbol{h}}$ can be expressed as

$$
\overrightarrow{\boldsymbol{h}}=\boldsymbol{U} \overrightarrow{e_{t}}+\boldsymbol{F}_{x} \overrightarrow{e_{x}}+\boldsymbol{F}_{y} \overrightarrow{e_{y}}+\boldsymbol{F}_{z} \overrightarrow{e_{z}}
$$

where (a) $\boldsymbol{U}$ represents the dependent conservative variables per unit spatial fluid volume; and (b) each of $\boldsymbol{F}_{x}, \boldsymbol{F}_{\mathrm{y}}$, and $\boldsymbol{F}_{z}$ represents the flux functions in the three spatial directions that are differentiable functions of $\boldsymbol{U}$ and its spatial derivatives in some cases. Then, the most fundamental and general form of the unsteady conservations laws applied over a space-time flow domain $D$ in $E_{4}$ can be cast into the following spacetime unity integral form:

$$
\oint_{\Omega} \vec{h} \cdot d \vec{s}=\int_{V} \boldsymbol{G} d V
$$

where the space-time flux vector is integrated over the surface $\Omega$ of an arbitrary space-time domain $V$ in $D$. The space-time surface area vector is defined as $d \vec{s}=\vec{n} d A$ where $\vec{n}$ is the outward surface unit normal and $d A$ is the space-time surface area increment in $\Omega$. The vector $\boldsymbol{G}$ is associated with possible source terms such as body force, chemical reaction, or other external forcing. For three-dimensional compressible Navier-Stokes equations, the dependent variables are defined by $\boldsymbol{U}=(\rho, \rho u, \rho v, \rho w, e)$ where $\rho, u, v, w$, and $e$ represent density, the three velocity components, and total energy per unit volume $\left(e=\frac{p}{\gamma-1}+\frac{\rho}{2}\left(u^{2}+v^{2}+w^{2}\right)\right)$, respectively. Flux vectors $\boldsymbol{F}_{x}, \boldsymbol{F}_{\mathrm{y}}$, and $\boldsymbol{F}_{z,}$ contain five elements to incorporate mass, three momentums, and energy conservation in the spatial coordinates $x, y$, and $z$, respectively. The source vector $\boldsymbol{G}$ is zero and the governing equations reduce to

$$
\oint_{\Omega} \overrightarrow{\boldsymbol{h}} \cdot d \vec{s}=0 .
$$

This form of the conservation law is valid for all 3D unsteady flows, including those with solution discontinuities (in space and time), such as shocks and contact discontinuities and is the form employed by the space-time CESE schemes.

Discretized equations of Eq. (3) for a tetrahedral element take the following form

$$
\sum_{i=1}^{N} \sum_{k=1}^{4} \overrightarrow{\boldsymbol{h}}_{i k} \cdot \Delta \vec{s}_{i k}=0 .
$$

In the CESE method, the individual space-time volume elements over which the space-time flux conservation is enforced is known as the conservation element $(\mathrm{CE})$ and its boundaries are part of what is 
denoted as a solution element (SE). Thereby, in Eq. (4), the index $i$ corresponds to one of $N$ CEs that make up the computational domain. For a tetrahedral element, there are: (i) a total of four neighboring CEs; and (ii) each CE is bound by five SEs (one corresponding to each of the element and its four neighbors). Within the SEs, the dependent variables $\boldsymbol{U}_{i}$ are assumed to be smooth and vary according to the Taylor series expansion,

$$
\boldsymbol{U}_{i}(x, y, z, t)=\boldsymbol{U}_{0 i}+\boldsymbol{U}_{t i}\left(t-t_{0}\right)+\boldsymbol{U}_{x i}\left(x-x_{0}\right)+\boldsymbol{U}_{y i}\left(y-y_{0}\right)+\boldsymbol{U}_{z i}\left(z-z_{0}\right)
$$

where $\left(x_{0}, y_{0}, z_{0}, t_{0}\right)$ is the solution point within the SE. The solution point is defined as the the centroid of all surrounding CEs. For high-order CESE schemes, Eq. (5) would contain higher derivatives(second and third for fourth-order schemes) in the Taylor series expansion.

In traditional finite-volume methods, the smoothness assumption is enforced within the control volume and the flow variable itself is assumed to be discontinuous across the boundaries of the control volume. In the CESE method by contrast, the smoothness assumption is only enforced at the boundaries of the $\mathrm{CE}$ and discontinuities are allowed to exist in the bulk of the space-time volume within the CE. With the boundaries of a $\mathrm{CE}$ being part of distinct solution elements, this approach allows for a unique definition of the flux leaving or entering each of the interfaces (using Eq. (5) without any reconstruction or Riemann approximation), thereby resulting in a genuinely multidimensional scheme. Additionally, in many of the common CFD algorithms, the temporal derivative is treated separately by using finite differences and spatial derivatives alone are integrated via either finite-volume or Galerkin methods. The CESE method, however, integrates the conservation laws over the entire discretized space-time domain in a unified manner. Such consistent formulation offers uniform temporal and spatial solution accuracy up to the designed order. For flow simulations, the conservation in both space and time has the potential to improve the temporal accuracy. More details of the numerical formulation used here can be found in Refs. 12 and 13.

\section{Code}

As mentioned in the previous section, the space-time CESE method is substantially different from conventional CFD software in many regards. The distinction between integration volume (CE) and solution volume (regions where the approximated polynomials are valid or SE) implies that the data structure has to allow divisions of mesh elements in two different ways. The space-time integration further requires constructions of general surfaces that extend over spatial and temporal coordinates simultaneously. The time-accurate local time-stepping scheme demands data structures to track partitions of each solution element and its proper communication with the neighbors that have a different number of partitions.

The NASA in-house CESE Navier-Stokes Solver, $e z 4 d$, has been designed and implemented from scratch to cope with these very different requirements that do not exist in existing CFD codes. For computational efficiency and ease of continual development, the ez $4 d$ software framework has been developed using a combination of object-oriented and generic programming paradigm in the $\mathrm{C}++$ programming language. Lightweight object-oriented hierarchy is used in conjunction with heavy use of template classes and functions to allow compile time polymorphism. Different conservation laws can be plugged in with templates that represent physics. Currently, the software supports either triangular/tetrahedral or quadrilateral/hexahedral unstructured meshes. Both multithread (based on lowlevel POSIX thread) and message passing interface (MPI) paradigms are used to facilitate large-scale parallel computations. Each MPI process within a computational node can be executed in multithread mode to further enhance parallel performance, especially for a memory bound multidomain layout.

Both second- and fourth-order CESE numerical schemes are implemented for general conservation laws including Euler and Navier-Stokes equations in the software framework. The TALTS scheme is used to enhance parallel performance for elements running at CFL numbers much smaller than one while maintaining a uniform temporal accuracy. Load balance for such runs can be improved via volume-weighted domain decomposition offered in the METIS $^{29}$ utility. For RANS simulations, implementations of the Sparlart-Allmaras and Mentor's SST-V models ${ }^{30}$ exist within the $e z 4 d$ framework. Large eddy simulations with subgrid scale dynamic-models capabilities have been recently added to the software for turbulent shear or boundary layer simulations. 


\section{Results and Discussion}

Results for the chosen test problems obtained using the $2^{\text {nd }}$-order accurate (time and space) CESE schemes and tetrahedral meshes are discussed in this section. The use of only $2^{\text {nd }}$-order accurate schemes in this paper is to assess feasibility of such methods in high-fidelity turbulent flow simulations.

\section{A. Canonical Isotropic Turbulence - Normal Shock Interaction}

The first problem under consideration concerns the most fundamental problem in shock/turbulence interaction, where complexities such as real-gas effects, nonuniform mean flow, etc., are ignored. Here, isotropic turbulence passes through a nominally normal shock in a perfect gas. A cubic domain of length $3 \pi$ in the streamwise direction and $2 \pi$ in the transverse directions was used in the computations. The normal shock was located at $x=\pi$. Unstructured meshes containing isotropic tetrahedrons generated with Pointwise $^{\circledR}$ are used for the solutions presented in this section. Depending on the strength of the shock and the intensity of the turbulence, two different regimes of interaction are possible, namely: (i) the wrinkled shock regime and (ii) the broken shock regime (Ref. 4). In the wrinkled shock regime, the shock retains its structure at all times, while it does not in the broken shock regime. Both regimes were examined as part of this work, by fixing the inflow turbulence intensity and varying the flow Mach number (shock strength). The $M_{1}=1.5$ and 1.87 conditions will correspond to the wrinkled shock regime, with $M_{1}$ being the Mach number upstream of the normal shock; and the $M_{1}=1.28$ condition corresponds to the broken shock regime.

The approach of imposing inflow conditions is adopted from earlier studies ${ }^{32-34}$ that specify the method for generating inflow turbulence tailored toward studying spatially-evolving turbulence and its interaction with a shock wave. Two important nondimensional parameters that characterize the state of the inflow are (i) turbulent Mach number, $M_{t}$, and (ii) Reynolds number based on the Taylor microscale, $R e_{\lambda}$. They are defined as follows:

$$
M_{t}=\frac{\sqrt{\left\langle u_{i} u_{i}\right\rangle}}{\langle c\rangle} ; R e_{\lambda}=\frac{\langle\rho\rangle u_{r m s} \lambda}{\langle\mu\rangle} \text {. }
$$

where,

$$
u_{r m s}=\sqrt{\frac{\left\langle u_{i} u_{i}\right\rangle}{3}} ; \quad \lambda^{2}=\frac{\left\langle u_{1}^{2}\right\rangle}{\left\langle\left(\partial u_{1} / \partial x\right)^{2}\right\rangle} .
$$

In the above equations, the symbol \langle\rangle stands for ensemble average and $\lambda$ represents the Taylor microscale. The flow field is initialized by setting up a random velocity field, $u_{i, 0}$ that is solenoidal and has an exponentially decaying velocity spectrum $E_{k}$, defined below, that corresponds to a specific initial $M_{t}$ and $R e_{\lambda}$.

$$
E(k) \sim k^{4} \exp \left(-2\left(k / k_{0}\right)^{2}\right) ; \frac{3 u_{r m s, 0}^{2}}{2}=\frac{\left\langle u_{i, 0} u_{i, 0}\right\rangle}{2}=\int_{0}^{\infty} E(k) d k .
$$

where, $k$ stands for the wavenumber magnitude. $k_{0}$, the most energetic wavenumber, is taken as 4 . For the chosen spectrum, the initial Taylor length scale $\lambda$ is $2 / k_{0}$. The initial density and pressure fields are assumed to be constant, and the remaining parameters are set based on $M_{t, 0}=0.22$ and $R e_{\lambda, 0}=19.9$. Periodic boundary conditions are used at the boundaries on the $y$ - and $z$-planes. At the outflow boundary, subsonic conditions corresponding to Rankine-Hugoniot jump conditions of the corresponding shock strength are used without a buffer domain. Previous studies ${ }^{4,32,33}$ of this problem needed special treatment of the outflow boundary condition via a buffer domain or sponge layer to damp any wave reflections and to further ensure that the shock remained stationary. No such special treatment of the outflow boundary condition was needed here. The simplicity and effectiveness of the outflow boundary conditions in the CESE method is an outcome of its foundational aspects. More details about it can found in Refs. 21, 34, and 35 . 
As part of the grid-sensitivity study, to assess the actual grid resolution required by the $2^{\text {nd }}$-order accurate CESE scheme for capturing the essential features of the flow, several isotropic meshes were tested. All of the meshes, with the exception of the finest mesh, did not have any clustering of mesh points around the shock region. The meshes without any packing of grids around the shock contained isotropic tetrahedrons throughout the domain with an edge length of approximately, $2 \pi / 64,2 \pi / 75,2 \pi / 96,2 \pi / 128$, and $2 \pi / 161$ resulting in a total element of approximately, 6, 9, 18, 33, and 52 Million tetrahedral cells, respectively. The finest mesh ( $\sim 66$ Million cells), see Fig. 1, contained cells with an approximate edge length of $2 \pi / 161$ in the coarse region and had cells packed in all three directions in a small region immediately behind the shock (with an approximate spacing of $2 \pi / 256$, that corresponds to approximately twice the shock thickness) to capture the rapid changes that occur due to the shock-turbulence interaction. The ability to pack grids only in a critical region without the grid distribution propagating to regions of lesser importance (as seen in simulations involving structured grids) is a primary advantage of using unstructured grids.

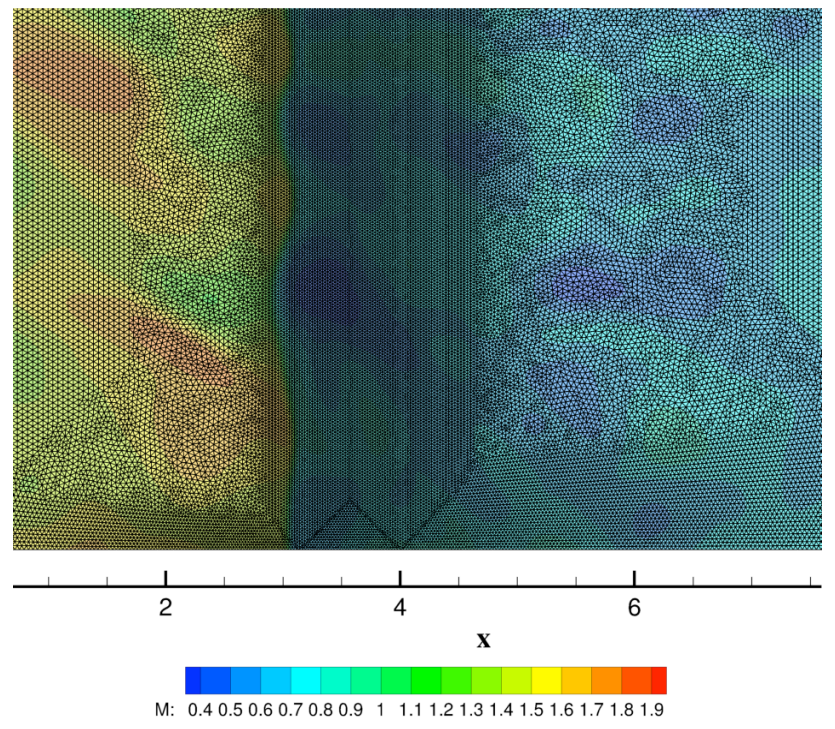

Figure 1. A slice of the mesh (one that includes packing of grids in the post-shock region) shaded with the local Mach number.

The instantaneous solution for the flow conditions of $\left(M_{t}=0.22, M_{1}=1.5\right)$ after approximately four flow-through times is shown in Fig. 2. The Q-criterion isosurfaces have been color shaded by the local Mach numbers to help distinguish the pre- and post-shock regions. Additionally, isosurfaces of dilatation contour are used in these plots to help visualize the instantaneous shock surface. The isotropic nature of the incoming turbulence is indicated by the random orientation of the vortex cores upstream of the shock surface. The shock compresses the turbulence in the streamwise direction, distorts the vortices, and in the meantime, makes them predominantly align in the $y-z$ plane (see the top view, Fig. 2). The weak distortion of shock surface seen here is representative of the wrinkled shock regime.

In all of the plots to be shown here onward, the streamwise coordinate has been nondimensionalized using the wavenumber of peak energy $k_{0}$, for facilitating comparison with linear theory. ${ }^{36}$ Furthermore, the nondimensionalized streamwise coordinates have been translated such that the shock is located at $x=0$. Averaging was performed over the transverse direction and in time by using the solution data collected over four flow-through times. For most of the conditions shown here, linear interaction analysis (LIA) is valid and the peak amplitudes predicted by it are provided for reference, wherever possible, along with additional computational results from Larsson and Lele. ${ }^{4}$ 


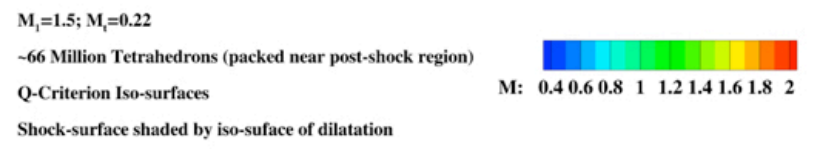

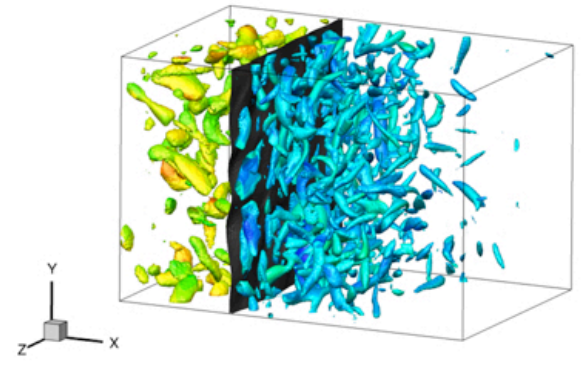

(a) Perspective View

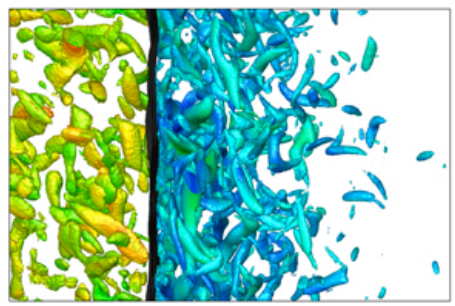

(b) Top View

Figure 2. Solutions for interaction of isotropic turbulence $\left(M_{t}=0.22\right)$ with a normal shock $\left(M_{1}=1.5\right)$, showing isosurface of $Q$-criterion (0.2) shaded with the local Mach number. Shock surface is represented by isosurface of dilatation (-1.0).

The flow condition corresponding to $M_{1}=1.5$ and $M_{t}=0.22$ was used for the grid convergence study. Vorticity, with its dependence on sensitive small-scale flow features, serves as a good indicator for analyzing grid convergence for this problem. Furthermore, it also brings out the underlying aspect that in the post-interaction region the initially isotropic turbulence becomes more anisotropic, due to the amplification of the transverse component of the vorticity. However, as can be seen from Fig. 3(a), anisotropy of the vorticity variance seems to indicate a rapid grid convergence. For this particular problem, the transverse component of the vorticity (normalized by the corresponding value upstream of the shock) undergoes a larger amplification than the streamwise vorticity. Therefore, looking into the amplification of the transverse component of the vorticity variance, Fig. 3(b), gives a better indication of how gridconverged the results are. With the two finest meshes used in the study, the amplification factor of the transverse vorticity obtained from the computations are close to that predicted by LIA ( 3.1, see Fig. 5(b) of Ref. 33) as well as computations of Larsson and Lele, ${ }^{4}$ indicative of nearing a grid converged solution. Although not shown here, evolution of the Reynolds stresses also indicates a rapid grid convergence, similar to the plot of vorticity anisotropy.

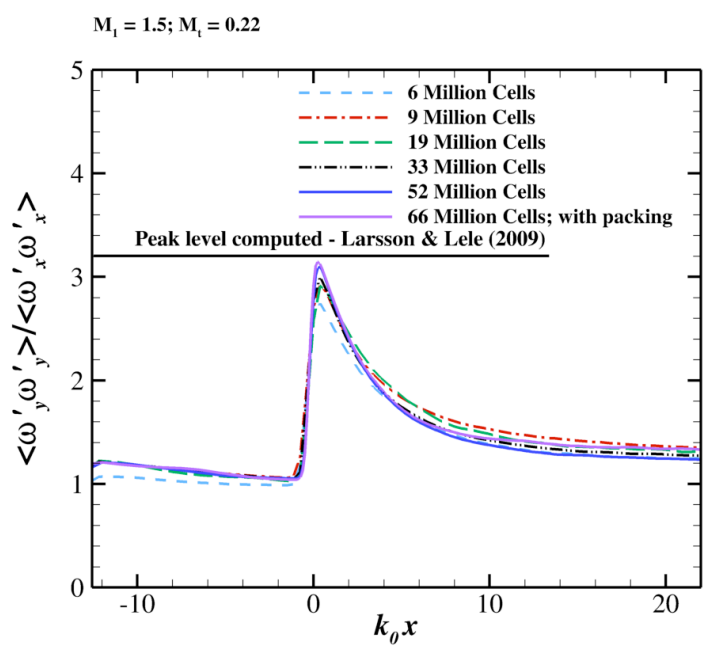

(a) Anisotropy of vorticity variance

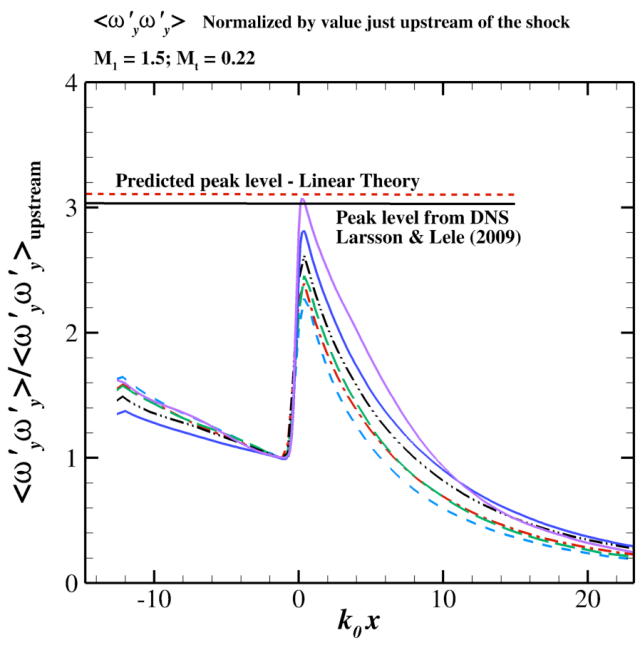

(b) Transverse vorticity variance

Figure 3. Evolution of vorticity variances obtained from the computations of normal shockturbulence interaction, using meshes with different resolutions. 
Given that the finest mesh captures the rapid amplification of vorticity in the vicinity of the postshock region correctly to the level predicted by LIA and other computations, it is indicative of the requirement of a finer grid resolution in the vicinity of the post-shock region. However, a closer look at the vorticity anisotropy plot (Fig. 3(a)) reveals that the vorticity is on its way to recovering its isotropy in regions beyond $k_{0} x \sim 15$, but the value is still above 1 . The failure to completely recover isotropy is indicative of a lack of adequate resolution in the regions closer to the outflow boundary, as suggested by Larsson and Lele, ${ }^{4}$ and needs to be investigated upon in the future with finer grid resolution in those regions.

All of the subsequent studies, focusing on the various shock turbulence interaction regimes, were carried out on the grid with 66 million cells that had packing of the grids in the vicinity of the post-shock region. The evolution of the streamwise and transverse components of the Reynolds stresses, along with the vorticity variances (normalized with their values just upstream of the shock), for various shock strengths are shown in Figs. 4(a)-(d). In general, for these flows at a given turbulent Mach number, the amplification of the Reynolds stresses and vorticity increases with an increase in shock strength at the region of interaction with the shock. The streamwise component of the Reynolds stress undergoes a rapid increase behind the shock before continuing to evolve nonmonotonically. The transverse stress component also increases at the shock, but then decays almost monotonically behind the shock. However, the level of fluctuation in the streamwise component just behind the shock is in general larger than the transverse component because of the contribution from the acoustic waves that are generated when the vortical waves interact with the shock. The post-shock increase of the streamwise component of the Reynolds stress is not predicted by the linear theory and can only be explained by nonlinear effects. ${ }^{42-33}$ A closer observation of Fig. 4(a) reveals that for the case of $M_{1}=1.28$ (red dashed-line curve) alone, the streamline component of the Reynolds stress does not see a secondary peak in the post-shock region and instead has a more monotonic decay. This is indicative of the broken shock regime of the interaction. Given the weak shock, the incoming turbulence level is significantly higher in this scenario compared to other conditions studied. The result is a stronger interaction where the shock gets broken creating many holes in the shock surface. Through these holes, the flow can pass through smoothly without experiencing any distortion. As a consequence, one does not see the nonlinear evolution of the transverse component of the stress in the postshock region and the general amplification of the transverse vorticity and Reynolds stresses are also comparatively small. The behavior of the Reynolds stresses observed in this study is consistent with what has been observed in the literature. ${ }^{4,32-33}$ As can be seen from Fig. 4(d), the predicted amplification rates of the transverse component of the vorticity for different Mach numbers from our computations compare well with that of LIA and computations of Larsson and Lele. ${ }^{4}$ Overall, the results indicate that the CESE method is able to capture the physics of the normal shock - turbulence interaction accurately on grids containing isotropic tetrahedral mesh elements.

\section{B. Oblique Shock - Turbulent Boundary Layer Interaction}

Flow of a Mach 2.9 supersonic turbulent boundary layer past a $24^{\circ}$ compression corner, the focus of experiments by Bookey et al. ${ }^{26}$ and Ringuette et al., ${ }^{27}$ is studied here using the CESE method and tetrahedral meshes. The same configuration has also been investigated using hexahedral meshes in other DNS studies. ${ }^{7}{ }^{28}$ The DNS study by $\mathrm{Wu}$ and Martin, ${ }^{7}$ was conducted concurrently along with the experiments and hence is also closer in conditions to the experiments. The goal here, in addition to validation of the computational tool, is to explore the flow physics from a different numerical perspective. The state of the incoming boundary layer in the experiment was $\theta=0.43 \mathrm{~mm}$; $\delta=6.7 \mathrm{~mm}$; and $C_{\mathrm{f}}=0.00225$. The reference flow conditions corresponding to the experimental study are given in Table 1. For all of the inflow computations performed in this section, periodic boundary conditions were enforced in the spanwise direction; solid walls were treated as being isothermal; and general nonreflecting boundary conditions were enforced at the outflow boundary. 


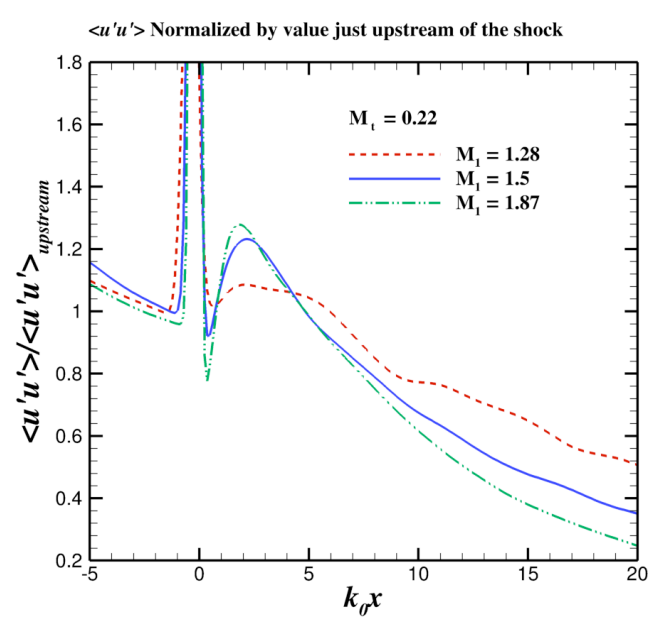

(a) Streamwise Reynolds stress component

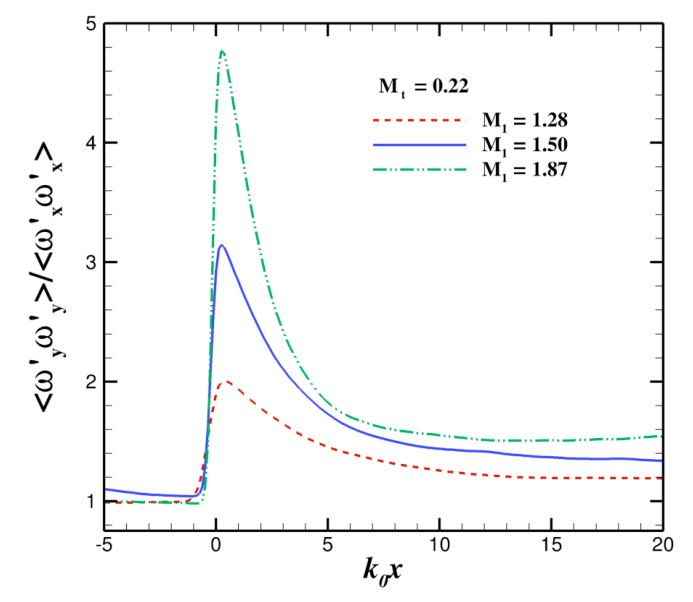

(c) Anisotropy of vorticity variance

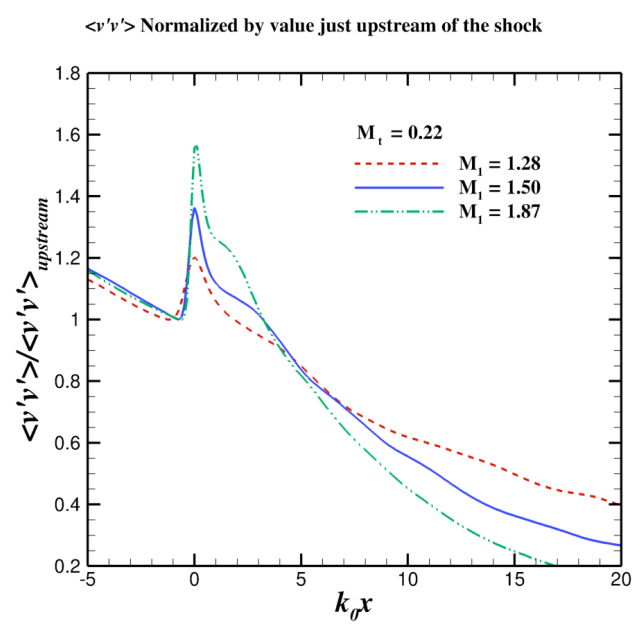

(b) Transverse Reynolds stress component

$\left.<\omega^{\prime} \omega_{y}^{\prime}\right\rangle$ Normalized by value just upstream of the shock

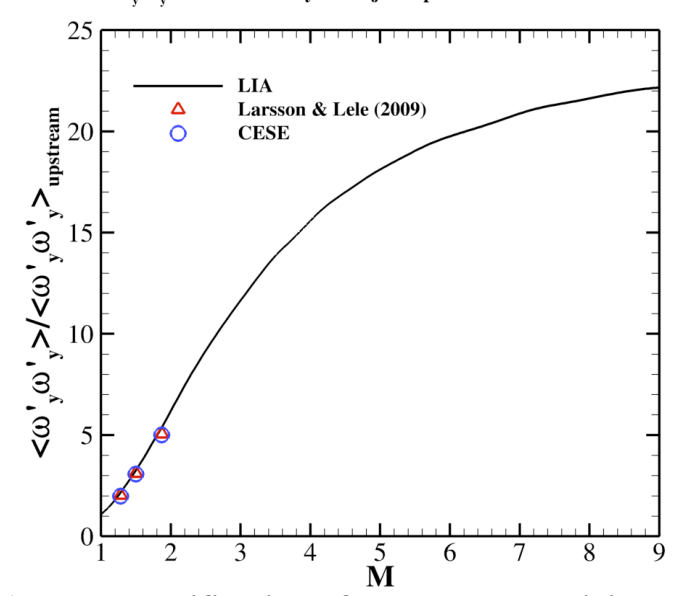

(d) Peak amplification of transverse vorticity variance

Figure 4. Evolution of vorticity variances and Reynolds stresses for different shock strengths.

Table 1. Reference flow conditions.

\begin{tabular}{cccccc}
\hline $\mathbf{M}$ & $\rho_{\infty}, \mathbf{k g} / \mathbf{m}^{\mathbf{3}}$ & $u_{\infty}, \mathbf{m} / \mathbf{s}$ & $T_{\infty}, \mathbf{K}$ & $\boldsymbol{R e}_{\mathbf{u n i t}}$ & $T_{\text {wall }} / T_{\text {adiabatic }}$ \\
\hline 2.9 & 0.0736 & 607.58 & 109.25 & $5.907 \times 10^{6}$ & 0.95 \\
\hline
\end{tabular}

\section{Inflow Turbulence Generation and its Assessment}

The ability to prescribe three-dimensional, time-dependent inflow boundary conditions is one of the most challenging and important aspects of performing DNS/LES of turbulent boundary layers. An effective approach can help substantially reduce the computational cost (smaller domain size) and also provide the correct physics without adding any artificial effects to the flow. A comprehensive survey of the various approaches is listed in Refs. 37 and 38. In this study, a variation of the rescaling-recycling method developed by Lund et al. ${ }^{39}$ is used to generate the incoming turbulent boundary layer. This approach requires an auxiliary computation wherein the desired inflow plane is located inside another computational box. This variation is similar to the method originally proposed by Spalart. ${ }^{40}$ The general procedure 
consists of the following key steps: (i) a periodic boundary condition is enforced in the streamwise as well as spanwise directions; (ii) the mean flow (obtained from RANS computations that match the desired inflow boundary layer conditions of the experiment) is frozen by adding source terms to the discretized governing equations, and (iii) the perturbations alone are recycled through the imposition of periodicity in the streamwise direction and their spatial growth is simulated by the governing equation. An initial random velocity perturbation field is generated assuming an isotropic turbulence spectra; and this field is then multiplied with a prescribed Reynolds stress tensor (estimated from experimental data or a previous computation), as proposed by Lund et al., ${ }^{39}$ to impose appropriate velocity RMS fluctuation distribution inside the boundary layer. Alternatively, a precomputed turbulent flow solution can also be used. The total initial field (mean plus perturbation) is evolved in time while the outflow perturbations are recycled into the inflow boundary. In general, the recycling simulation will settle down with a dynamic turbulent flowfield after about 2-3 flow through times, after the rather arbitrary initial fluctuation field has been "washed out" by the time-accurate Navier-Stokes solver. The prescribed Reynolds stress tensor used here was obtained from the supersonic turbulent boundary layer database ${ }^{*}$ maintained by Prof. Sergio Pirozzoli's research group. The strong Reynolds analogy (SRA) is used to prescribe the corresponding fluctuations in the thermodynamic variables. SRA relates fluctuations in thermodynamic variables to the previously determined velocity fluctuations. To prevent spanwise locking of large-scale structures, an outcome of the imposition of periodicity in streamwise directions, the spanwise shifting approach developed by Munters et al. ${ }^{41}$ is also used.

For the above recycling computations (towards inflow generation), a smaller domain of size $6 \delta_{0}$ in the streamwise direction, $5 \delta_{0}$ in the wall-normal direction and $3 \delta_{0}$ in the spanwise direction was used. The mesh used was obtained by slicing a hexahedral mesh into tetrahedrons. Isotropic tetrahedral elements were not used in this problem to avoid the large grid count that would result from trying to resolve the boundary layer down to the $y^{+}=1$ range. The hexahedral mesh was uniformly spaced in streamwise and spanwise directions, with geometric stretching applied in the wall normal direction. After 2-3 domain flow through times, when all transient effects have been washed out, the time-series is collected on points lying in a plane (representative of the inflow boundary of the main ramp computation) that is located midway in the streamwise direction. In other words, this unsteady data serves as the time-dependent boundary condition for the DNS computation.

To assess the quality of the inflow generation approach, the data was fed into the flat plate section of the ramp configuration using the grid with the finest resolution (described in the following section) to simulate a spatially evolving Mach 2.9 zero-pressure gradient boundary layer. As shown by the Q-criteria plot in Fig. 5(a), the flow evolves in the streamwise direction revealing alternating hairpin vortex structures near the outer (free-stream side) part of the boundary layer. Additional smaller structures closer to the wall are also evident. The mean flow characteristics (not shown here) also appear to be correctly captured. Fig. 5(b) shows the velocity fluctuation and temperature fluctuation profiles at three different streamwise locations. The near-wall peak of the streamwise velocity fluctuations appears to grow with distance from the inflow plane (increase in Reynolds number), while the wall-normal velocity fluctuations appear to take on a plateau like shape with intensity levels lower than that of the streamwise velocity. As for the temperature, a substantial level of fluctuations persists away from the wall. The overall level of the fluctuations and the location at which the intensities peak are in agreement with those reported in the literature. ${ }^{7,42}$ As no scaling (van Driest) has been applied to the turbulence intensity data, the data appear to be scattered and do not collapse. These fluctuation profiles are similar in shape as the turbulent boundary layer evolves downstream of the plate without any observable transient effects from the inflow plane (where the solution from recycling simulation are fed in). This observation provides good evidence that the recycling simulation solution represents the turbulent boundary layer properly at the prescribed Reynolds number

\footnotetext{
${ }^{*}$ http://reynolds.dma.uniroma1.it/dnsm2/
} 


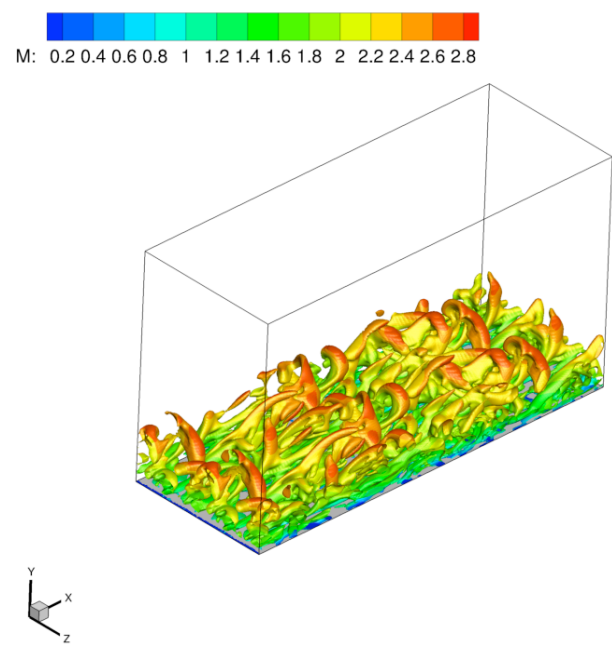

(a) Q-criterion shaded by Mach contour

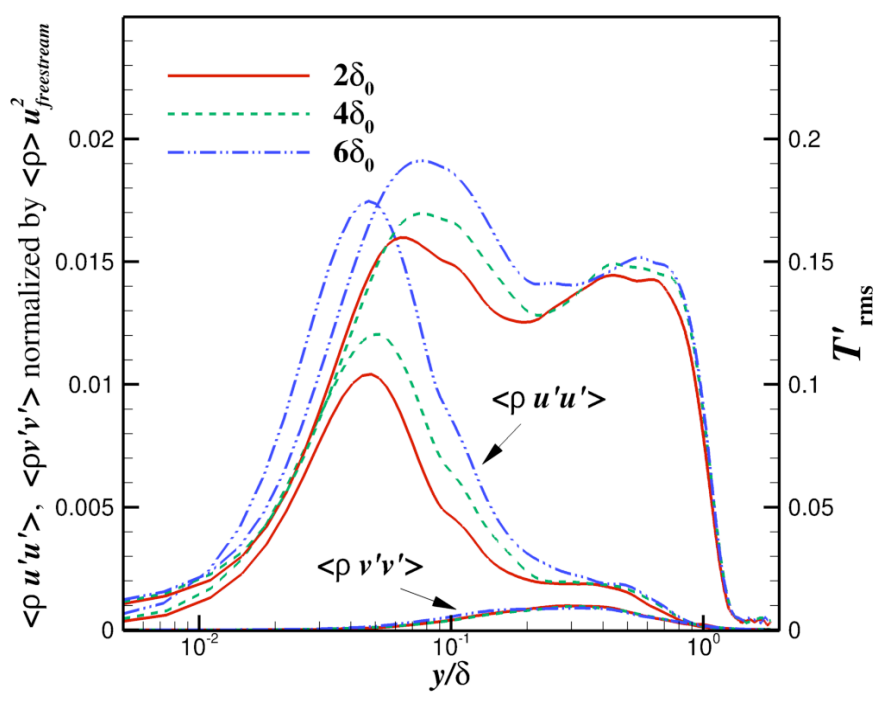

(b) Statistics of velocity and temperature fluctuations

Figure 5. A spatially evolving Mach 2.9 turbulent boundary layer visualized through its $Q$-criterion and the statistics of its fluctuations.

\section{Grid Sensitivity}

In any DNS study mesh resolution is expected to be important; even more so here given the interplay between shocks and turbulent boundary layer present in the flow. Three different grids were generated to ascertain its sensitivity on the solution. The mesh was obtained by slicing a structured mesh (hexahedral elements). The hexahedral mesh was uniformly spaced in the streamwise and spanwise directions (without any packing toward the corner) and employed geometric stretching in the wall normal direction. Complete details about the domain size and mesh spacing are given in Table 2. Given the simplicity of the geometry and the importance of the boundary layer region to the flow, the above-mentioned mesh generation approach (slicing a structured mesh) was the easiest to ensure that sufficient grid points (in the wall-normal direction) are within the boundary layer. Alternatively, one could ensure that the necessary wall-normal mesh spacing is met through the use of anisotropic grid generation features in commercial grid generation codes (e.g., the T-Rex feature of Pointwise ${ }^{\circledR}$ ). Such an approach will be adopted in the future to benefit from the added ability that truly unstructured grids provide in terms of ability to pack or adapt grids in the relevant regions around the shock.

Table 2. Details for the grid sensitivity study. The distance in the streamwise direction is broken into section lengths upstream and downstream of the corner.

\begin{tabular}{cccccc}
\hline Mesh & $L_{x} \times L_{y} \times L_{z}\left(\right.$ in $\left.\delta_{0}\right)$ & $\Delta y^{+}$ & $\Delta x^{+}$ & $\Delta z^{+}$ & $\begin{array}{c}\text { No. Cells } \\
\text { (tetrahedrons) }\end{array}$ \\
\hline Mesh 1 & $(8+6) \times 5 \times 3$ & 0.4 & 12 & 8 & $\sim 15$ Million \\
Mesh 2 & $(8+6) \times 5 \times 3$ & 0.4 & 8 & 4 & $\sim 25$ Million \\
Mesh 3 & $(8+5) \times 5 \times 3$ & 0.2 & 6 & 3 & $\sim 45$ Million \\
\hline
\end{tabular}

As shown in Fig. 6(a), the spanwise-averaged mean (time-averaged) velocity profiles at the inflow plane appear to collapse on top of each other for the various mesh resolutions, but significant discrepancies are seen in the mean wall pressure distribution plot shown in Fig. 6(b). The coarser meshes (Mesh 1 and Mesh 2), predict much earlier separation as compared to that of experiment, while results from Mesh 3 appears to come closest toward matching the experimental results (more details in a subsequent section). As indicated by Fig. 7, Mesh 2 is able to capture the general features of the flow, such as (i) the separation and reattachment shocks; (ii) the series of compression waves at the root of the shock, seen from the 
numerical schlieren contour plot (exponential function of density gradient magnitude as proposed by $\mathrm{Wu}$ and $\operatorname{Martin}^{7}$ ); and (iii) the hairpin vortices changing from their long, and thin forms that are aligned in the streamwise directions into a more shorter post-shock structure that is inclined to the wall, in the ramp section. However, as indicated by the mean pressure plot, better agreement in mean wall pressure comes only with an increase in spanwise and wall-normal resolution. A streamwise grid clustering near the corner of the ramp could potentially improve the resolution of the separation region length, a topic for future studies. Another possible reason for this discrepancy could be due to a mismatch in the boundary layer momentum thickness (discussed in the subsequent section) as compared to the experiments.

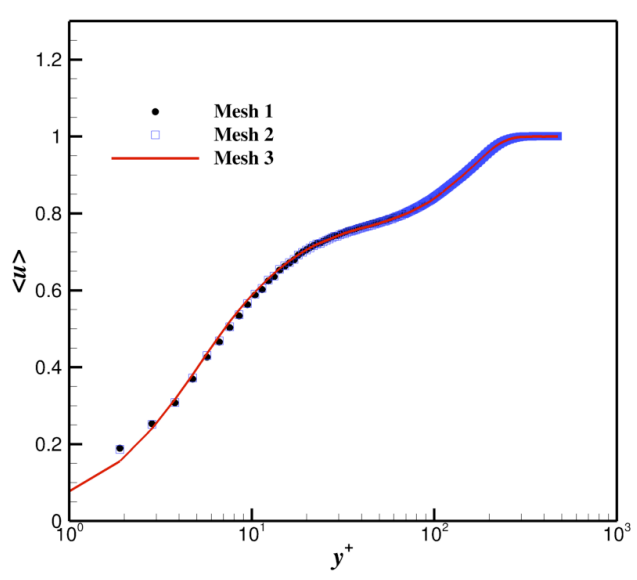

(a) Velocity profile at inflow

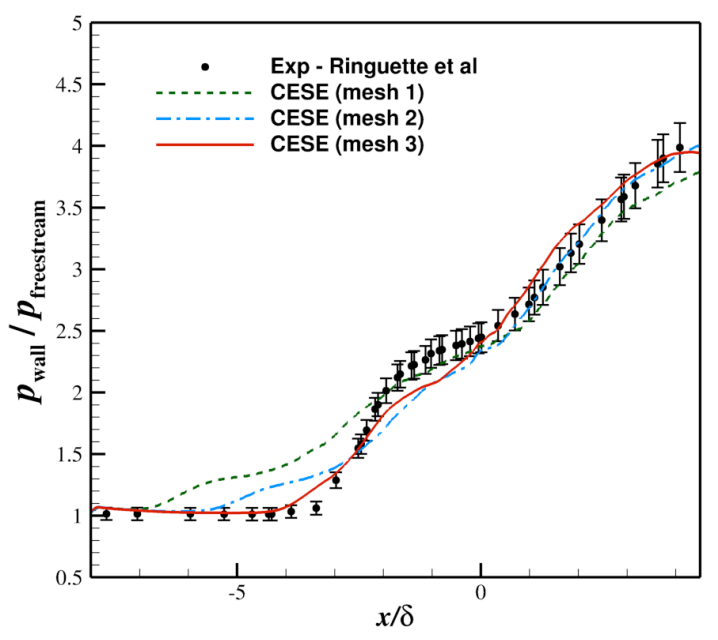

(b) Mean wall pressure distribution

Figure 6. Influence of mesh resolution on the mean properties of the flow.

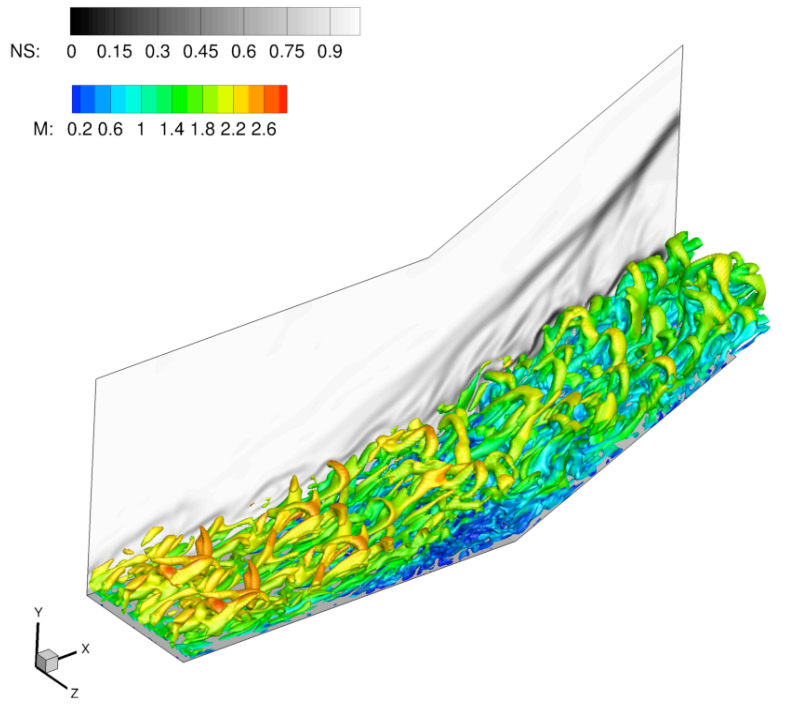

Figure 7. Isosurfaces of Q-criterion (1000) shaded by Mach number and a z-plane cut shaded by the numerical schlieren contours are shown for the computation performed using Mesh 2.

\section{Statistics and accuracy of the computation}

Results reported in this section were obtained using Mesh 3. Statistics were collected over 6 domain flow through times, i.e., the time for the free stream to travel about 70 times the inflow boundary layer thickness. This time period may not be able to track the very low frequency mode that may exist, but it is 
generally deemed to be enough for the quantities whose statistics are reported below (in Ref. [7], statistics were collected over a period equivalent to that needed by the freestream to travel 300 times the boundary layer thickness). An instantaneous plot of the shock surface and a numerical schlieren contour of a spanwise plane are shown in Figs. 8 (a) and (b) as a demonstration of CESE's ability to accurately capture the flow details using tetrahedral elements (even within the boundary layer). Features such as the bending of the main shock as well as the weak shocklets that emerge from the edge of the boundary layer in the ramp section of the flow can be easily discerned from Fig. 8(b).

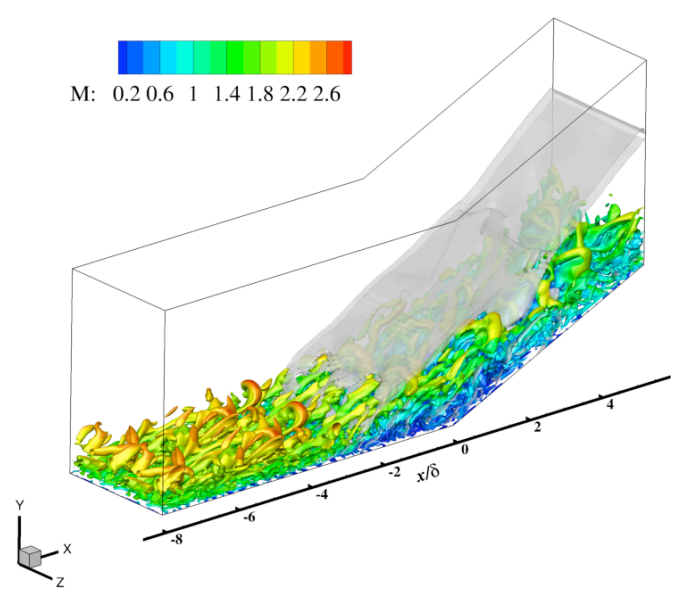

(a) Isosurfaces of Q-criterion and dilatation contour (shock surface)

Figure 8. Instantaneous shock surface and density gradient contours of the 2.9 turbulent boundary layers flow over a compression corner.

Table 3. Comparison of relevant parameters in the simulation against available data.

\begin{tabular}{lccc}
\hline Quantity & Experiment $^{\mathbf{2 6 , 2 7}}$ & DNS (Wu and Martin) & DNS (CESE) \\
\hline Wall temperature $(\mathrm{K})$ & 260.24 & 307.0 & 260.24 \\
$C_{f}$ & 0.00225 & 0.00217 & 0.00209 \\
$\delta_{0}(\mathrm{~mm})$ & 6.7 & 6.4 & 6.7 \\
$\theta_{0}(\mathrm{~mm})$ & 0.43 & 0.38 & 0.49 \\
$\operatorname{Re}_{\theta}$ & 2400 & 2300 & 2900 \\
Separation point & $-3.2 \delta_{0}$ & $-3.0 \delta_{0}$ & $-3.5 \delta_{0}$ \\
Reattachment point & $1.6 \delta_{0}$ & $1.2 \delta_{0}$ & $1.4 \delta_{0}$ \\
\hline
\end{tabular}

Flow parameters of interest, obtained from the mean flow, are compared with experiments and other DNS results in Table 3. In the current computation, the initial momentum thickness of the boundary layer appears to be larger than that found in the experiments, but other parameters such as the boundary layer thickness and skin friction coefficient are close to the measured data. In general, Fig. 9(a) shows the discrepancies between the current inflow profile and the data to be within the error bar for $y / \delta>0.3$. Further inside the boundary layer, there are slight discrepancies between current results and data from the DNS study of $\mathrm{Wu}$ and Martin, ${ }^{7}$ possibly reminiscent of discrepancies in boundary layer (momentum) thickness. More studies are needed to identify the differences. Based on mean skin friction coefficient data, separation and reattachment points were determined and compared with data in Table 3. The predicted separation location is slightly earlier than that found in the experiment, but the overall (near-wall) separation region measured at the wall matches closely. Figure 9(b) shows the wall parallel velocity profile at $x=4 \delta_{0}$ downstream of the corner. The overall agreement is reasonably good with most data points falling within the $5 \%$ uncertainty in experimental measurements. It is interesting to observe that there is better agreement between the current investigation and Ref. 7 in the downstream region than upstream (Fig. $9(\mathrm{a}))$. 


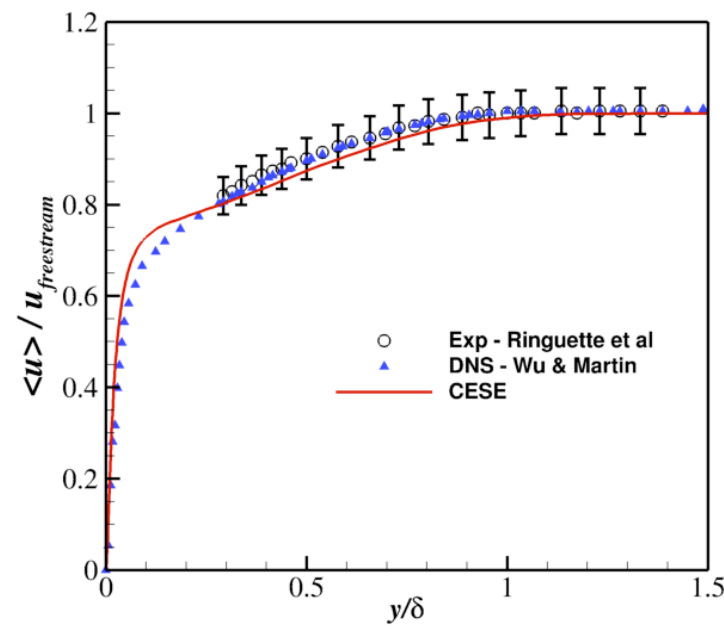

(a) Velocity profile at inflow (normalized by free stream value)

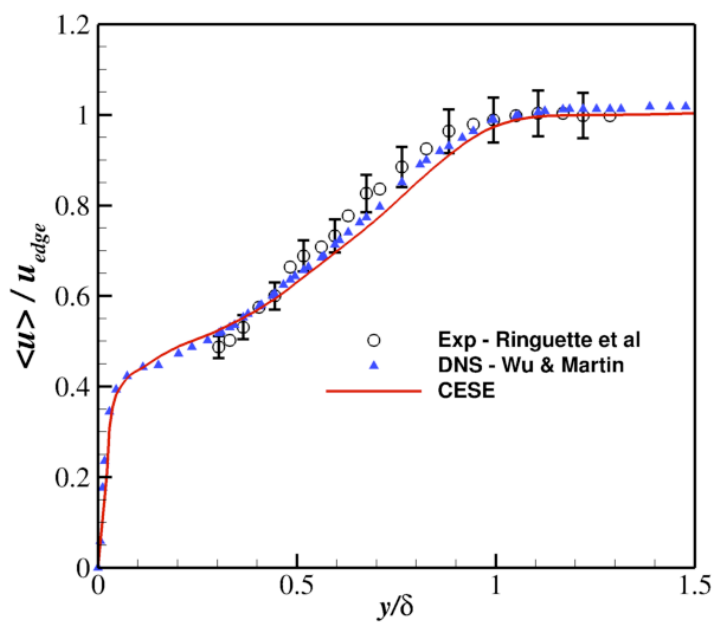

(b) Wall parallel velocity profile $4 \delta_{0}$ downstream of the corner (normalized by boundary layer edge value)

Figure 9. Velocity profiles at two stations compared against experimental and available DNS data.

The mean wall pressure distribution is one of the key features of the SWBLI problem. Depending on the ramp angle, the pressure distribution varies significantly. The distinctive pressure plateau that is indicative of the separation region begins to appear for ramp angles beyond a certain threshold value. ${ }^{43}$ Comparison of the mean wall pressure distribution obtained from the current simulation against available data is shown in Fig. 10(a). Earlier separation and reattachment locations reported previously in this section is evident from the rise in pressure that appears to happen around $x=-4 \delta_{0}$. The plateau portion of the pressure distribution also appears to terminate earlier, resulting in a lower level of pressure within the plateau portion of the curve. However, the recovery of the pressure in the post-shock region to its inviscid value does appear to follow the experimental data as well the DNS data of $\mathrm{Wu}$ and Martin. ${ }^{7}$ Previous studies ${ }^{7,44}$ have indicated the presence of a large scale, slow motion of the shock that makes the separation bubble oscillate with a very low frequency. However, the statistics collected in this study was not sufficiently long enough to account for the low-frequency effect. The wall pressure fluctuations in terms of the normalized RMS values are shown in Fig. 10(b). Data from the experiments of Bookey et al. ${ }^{26}$ and other DNS computations ${ }^{7,28}$ have also been plotted for comparison. The pressure fluctuation appears to show two peaks along the wall, one upstream of the corner inside the separation region and one downstream in the reattached region. The second peak from the current investigation, unlike that from $\mathrm{Wu}$ and Martin, ${ }^{7}$ is larger than the first peak similar to the results of Muppidi and Mahesh. ${ }^{28}$ The overall wall pressure fluctuations in the current study appear to be somewhere in the middle between those found in the other two DNS results. The second peak observed here could be associated with the focusing effects coming from the interaction of turbulent structures with the compression waves formed atop the separation region. However, the experimental data only shows a relatively mild growth in wall pressure fluctuations on the ramp. All three computational results overpredict the wall pressure fluctuations in general but to a different extent. The reason for higher computational pressure fluctuations is unknown and could be a topic for future studies. 


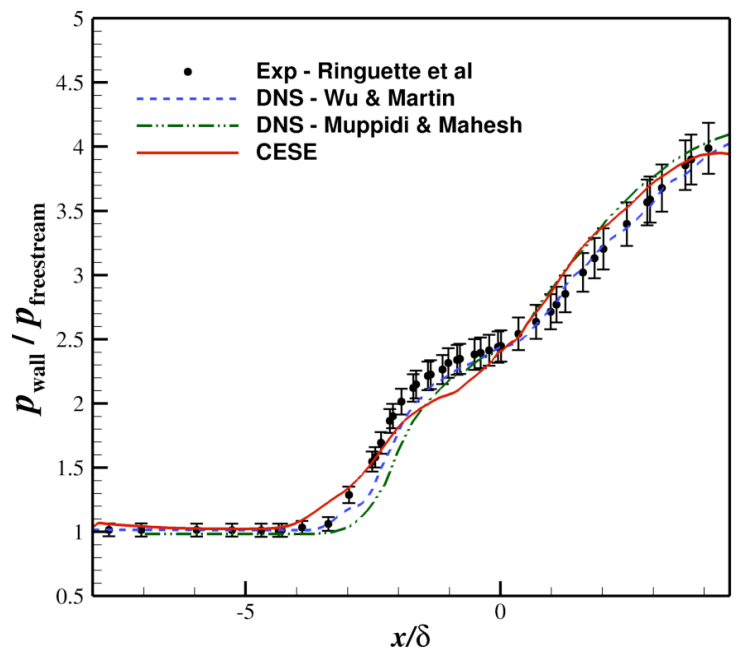

(a) Mean wall pressure distribution

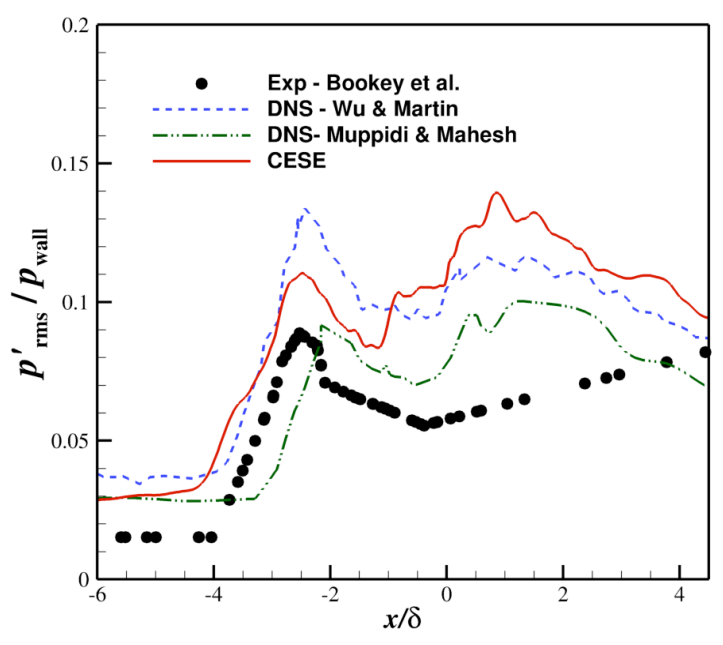

(b) Normalized wall pressure fluctuation

Figure 10. Comparison of mean wall pressure and its fluctuation, obtained from present simulation, against other available data.

Figure 11 shows the spanwise averaged mean velocity contour along with the streamwise locations at which the various components of the Reynolds stress as well as the mass-flux turbulence intensity, shown in Fig. 12, have been extracted to be analyzed. As can be seen from Figs. 12 (a)-(c), the various components of the Reynolds stresses are generally amplified downstream of the interaction region, similar to what was seen in the case of isotropic turbulence passing through a normal shock (Section IV. (A)). The $\rho v^{\prime} v^{\prime}$ and $\rho u^{\prime} v^{\prime}$ components in general appear to experience larger amplification than the streamwise component. The overall levels appear to be smaller than those found in the DNS studies by Wu and Martin, ${ }^{7}$ but the trend closely mirrors those found in that study as well as others. ${ }^{28,}{ }^{45}$ Given that massflux turbulence amplification is a direct function of the pressure rise, the levels downstream of the interaction (see Fig. 12(d)) are much higher than those before it and the overall amplification factor of approximately 5 is in line with those predicted by other studies. $7,45,46$

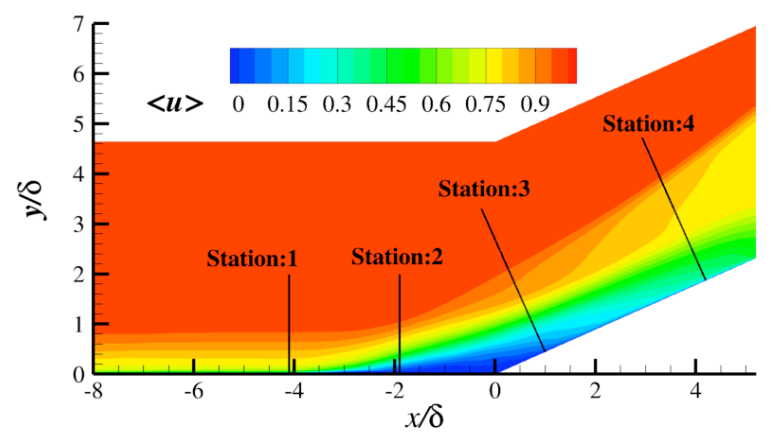

Figure 11. Mean velocity contour is shown along with locations at which Reynolds stress data have been extracted. 


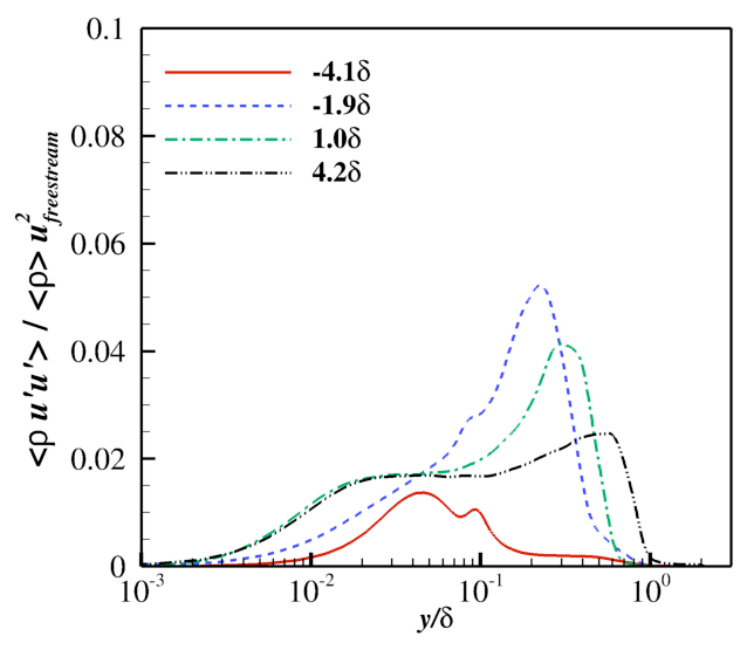

(a) $\rho u^{\prime} u^{\prime}$

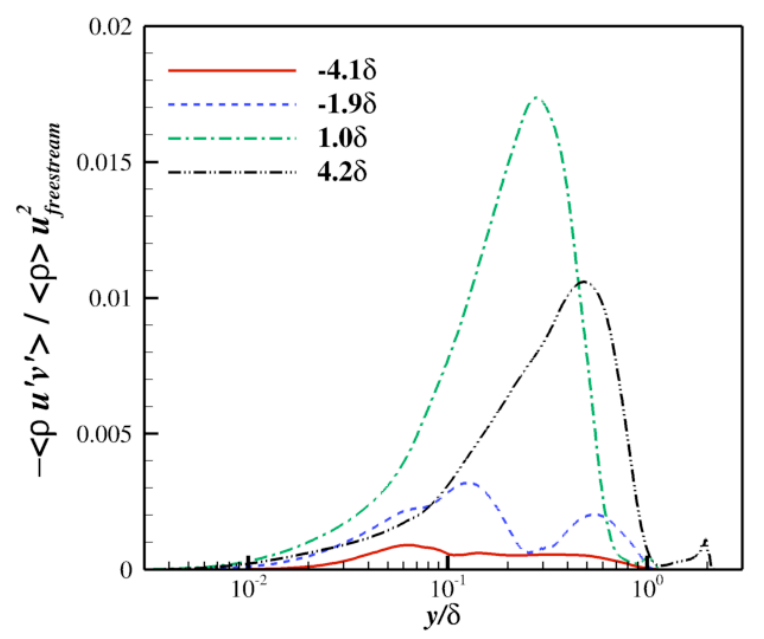

(c) $-\rho u^{\prime} v^{\prime}$

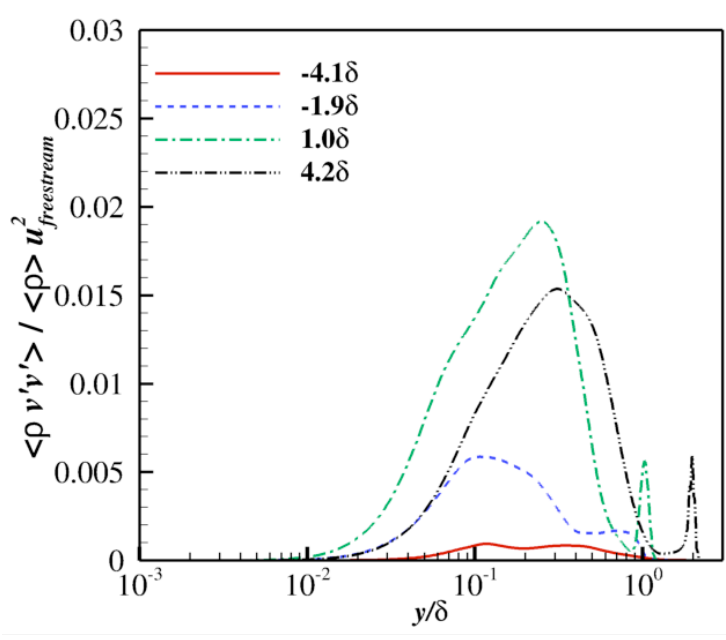

(b) $\rho v^{\prime} v^{\prime}$

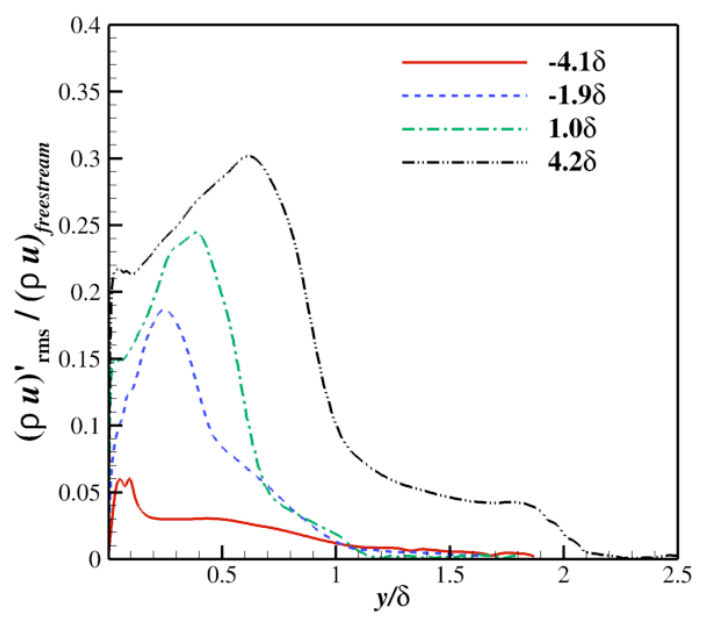

(d) Mass-flux turbulence intensity

Figure 12. Reynolds stress and mass-flux turbulence intensity at various streamwise locations.

\section{Summary}

Application of the novel space-time CESE numerical method for computing shock-turbulence interactions with tetrahedral meshes was reported. For the case of a normal shock interacting with isotropic turbulence, both the regimes of wrinkled shock and broken shock were investigated. Grid convergence was demonstrated for the regime of a wrinkled shock. Overall, the various modes through which the turbulence interacts with the normal shock and the associated flow physics is accurately captured by CESE and was found to be consistent with both linear theory as well as previous DNS investigations. DNS-accessible turbulent shock boundary-layer interaction was also investigated as part of this work. To the knowledge of the authors, tetrahedral meshes were used for the first time in DNS studies of this particular SWBLI problem. The necessary time-dependent inflow boundary data were generated using an auxiliary computation that followed the recycling approach. The results indicate reasonable agreement with existing experimental data as well as DNS results. Some discrepancies in the incoming boundary layer momentum thickness as well as in the mean wall pressure distribution were observed. The differences in the inflow turbulent boundary layers among different DNS results could play a role. The exact cause of these discrepancies is left for future studies. A longer integration time is also needed in future work to account for the low-frequency separation bubble oscillation. In summary, the overall results for both canonical problems indicate that the CESE method is a promising alternative to conduct DNS studies on complex configurations and flows involving shock-turbulence interactions with pure unstructured meshes. 


\section{Acknowledgments}

This research was sponsored by the NASA Transformational Tools and Technologies (TTT) project of the Transformative Aeronautics Concepts Program under the Aeronautics Research Mission Directorate. The first author's research is funded through the National Institute of Aerospace (NIA) under the cooperative agreement 2A00. Resources supporting this work were provided by the NASA High-End Computing (HEC) Program through the NASA Advanced Supercomputing (NAS) Division at Ames Research Center. The authors would also like to thank Dr. Prahladh Iyer of NIA for valuable technical exchanges.

\section{References}

1. Bermejo-Moreno, I., Bodart, J., Larson, J., Barney, B. M., Nichols, J. W., and Jones, S., "Solving the Compressible Navier-Stokes Equations on up to 1.97 Million Cores and 4.1 Trillion Grid Points," in Proceedings of the 2013 ACM/IEEE Conference on Supercomputing (SC13), 2013.

2. Candler, G., Barnhardt, M., Drayna, T., Nompelis, I., Peterson, D., and Subbareddy, P., "Unstructured Grid Approaches for Accurate Aeroheating Simulations," AIAA Paper 2007-3959, 2007.

3. Gnoffo, P. A., "Multi-Dimensional, Inviscid Flux Reconstruction for Simulation of Hypersonic Heating on Tetrahedral Grids," AIAA Paper 2009-0599, 2009.

4. Larsson, J., and Lele, S. K., "Direct Numerical Simulation of Canonical Shock/Turbulence Interaction," Phys. Fluids, Vol. 21, No. 12, 2009, pp. 126101

5. Sivasubramanian, J., and Fasel, H. F., "Numerical Investigation of Shock-Induced Laminar Separation Bubble in a Mach Boundary Layer," AIAA Paper 2015-2641, 2015.

6. Pirozzoli, S., and Grasso, F., "Direct Numerical Simulation of Impinging Shock Wave/Turbulent Boundary Layer Interaction at M=2.25," Phys. Fluids, Vol. 18, No. 6, 2006, pp. 65113.

7. Wu, M., and Martin, M. P., "Direct Numerical Simulation of Supersonic Turbulent Boundary Layer over a Compression Ramp," AIAA J, Vol. 45, No. 4, 2007, pp. 879-89.

8. Lele, S. K., "Compact finite difference schemes with spectral-like resolution,” J. Comput. Phys., Vol. 103, 1992, pp. 16-42.

9. Balsara, D., and Shu, C.-W., "Monotonicity preserving weighted essentially non-oscillatory schemes with increasingly high order of accuracy," J. Comput. Phys., Vol. 160, 2000, pp. 405-452.

10. Larsson, J., Lele, S. K., and Moin, P., "Effect of Numerical Dissipation on the Predicted Spectral for Compressible Turbulence," in Annual Research Briefs (Center for Turbulence Research, CA), 2007, pp. 47.

11. Khalighi, Y., Nichols, J. W., Lele, S. K., Ham, F., and Moin, P., "Unstructured Large Eddy Simulation for Prediction of Noise Issued from Turbulent Jets in Various Configurations," AIAA Paper 2011-2886, 2011.

12. Chang, S.-C., "The Method of Space-Time Conservation Element and Solution Element-A New Approach for Solving the Navier-Stokes and Euler Equations," J. Comput. Phys., Vol. 119, 1995, pp. 295-324.

13. Chang, S.-C., "A New Approach for Constructing Highly Stable High Order CESE Schemes," AIAA Paper 2010-543, 2010.

14. Chang, C.-L., "Three-Dimensional Navier-Stokes Calculations Using the Modified Space-Time CESE Method," AIAA Paper 2007-5818, 2007.

15. Chang, C.-L., Venkatachari, B., and Cheng, G, "Time-Accurate Local Time Stepping and High-Order SpaceTime CESE Methods for Multi-Dimensional Flows with Unstructured Meshes," AIAA Paper 2013-3069, 2013.

16. Chang, S.-C., Wu, Y., Yang, V., and Wang, X.-Y., "Local Time-Stepping Procedures for the Space-Time Conservation Element and Solution Element Method," Inter. J. of Comput. Fluid Dyn., Vol. 19, No. 5, 2005, pp. 359-380.

17. Yen, J. C., "Demonstration of a Multi-Dimensional Time-Accurate Local Time Stepping CESE Method," AIAA Paper 2011-2755, 2011.

18. Chang, C.-L., Choudhari, M., and Li, F., "Numerical Computations of Hypersonic Boundary-Layer over Surface Irregularities," AIAA Paper 2010-1572, 2010.

19. Chang, C.-L., Choudhari, M., Li, F., and Venkatachari, B. S., "Effects of Cavities and Protuberances on Transition over Hypersonic Vehicles,” AIAA Paper 2011-3245, 2011. 
20. Moin, P., and Mahesh, K., "Direct Numerical Simulation: A Tool in Turbulence Research," Annu. Rev. Fluid Mech., Vol. 30, 1998, pp. 539-578.

21. Chang, C.-L., Venkatachari, B. S., and Cheng, G. C., "Tetrahedral-Mesh Simulation of Turbulent Flows with the Space-Time Conservative Schemes," AIAA Paper 2015-3084, 2015.

22. Dolling, D. S., "Fifty Years of Shock-Wave/Boundary-Layer Interaction Research: What Next?," AIAA J., Vol. 39, No. 8, 2001, pp. 1517-1531.

23. Knight, D., Yan, H., Panaras, A.G., and Zheltovodov, A., "Advances in CFD Prediction of Shock Wave Turbulent Boundary Layer Interactions,” Prog. Aero. Sci., Vol. 39, 2003, pp. 121-184

24. Edwards, J. R., "Numerical Simulations of Shock/Boundary Layer Interactions Using Time-Dependent Modeling Techniques: A Survey of Recent Results," Prog. Aero. Sci., Vol. 44, 2008, pp. 447-465.

25. Gaitonde, D. V., "Progress in Shock Wave/Boundary Layer Interactions," Prog. Aero. Sci., Vol. 72, 2015, pp. $80-99$.

26. Bookey, P. B., Wyckham, C., Smits, A. J., and Martin, M. P., "New Experimental Data of STBLI at DNS/LES Accessible Reynolds Numbers," AIAA Paper 2005-309, 2005.

27. Ringuette, M. J, Bookey, P. B., Wyckham, W., and Smits, A. J., "Experimental Study of a Mach 3 Compression Ramp Interaction at $\mathrm{Re}_{\theta}=2400$," AIAA J., Vol. 47, No. 2, 2009, pp. 373-385.

28. Muppidi, S., and Mahesh, K., "DNS of Unsteady Shock Boundary Layer Interaction," AIAA Paper 2011724, 2011.

29. Kumar, V., and Karypis, G., "A Fast and High Quality Multilevel Scheme for Partitioning Irregular Graphs," SIAM J. Sci. Comp., Vol. 20, No. 1, 1999, pp. 359-392.

30. "Turbulence Modeling Resource," NASA Langley Research Center, URL: http://turbmodels.larc.nasa.gov [cited 19 Nov, 2017].

31. Lee, S., Lele, S. K., and Moin, P., "Simulation of Spatially Evolving Turbulence and the Applicability of Taylor's Hypothesis in Compressible Flow," Phys. Fluids A, Vol. 4, No. 7, 1992, pp. 1521-1530.

32. Lee, S., Lele, S. K., and Moin, P., "Direct Numerical Simulation of Isotropic Turbulence Interacting with a Weak Shock Wave," J. Fluid Mech., Vol. 251, 1993, pp. 533-562.

33. Lee, S., Lele, S. K., and Moin, P., "Interaction of Isotropic Turbulence Interacting with Shock Waves: Effect of Shock Strength," J. Fluid Mech., Vol. 340, 1997, pp. 225-247.

34. Loh, C. Y., Hultgren, L. S., and Chang, S.-C., "Wave Computation in Compressible Flow Using Space-Time Conservation Element and Solution Element Method," AIAA J., Vol. 39, No. 5, 2001, pp. 794-801.

35. Chang, S.-C., Himansu, A., Loh, C. Y., Wang, X.-Y, and Yu, S.-T., "Robust and Simple Non-Reflecting Boundary Conditions for the Euler Equations-A New Approach Based on the Space-Time CE/SE Method," NASA/TM-2003-212495, 2003.

36. Anyiwo, J. C., and Bushnell, D. M., "Turbulence Amplification in Shock-Wave Boundary Layer Interaction," AIAA J., Vol. 20. No. 7, 1982, pp. 893-899.

37. Dhamankar, N. S., Blaisdell, G. A., and Lyrintzis, A. S., "An Overview of Turbulent Inflow Boundary Conditions for Large Eddy Simulations," AIAA Paper 2015-3213, 2015.

38. Wu, X., "Inflow Turbulence Generation Methods," Annu. Rev. Fluid Mech., Vol. 49, 2017, pp. $23-49$.

39. Lund, T. S., Wu, X., and Squires, K. D., "Generation of Turbulent Inflow Data for Spatially-Developing Boundary Layer Simulations,” J. of Comput. Phys, Vol. 140, 1998, pp. 233-258.

40. Spalart, P. R., "Direct Simulation of a Turbulent Boundary Layer up to $\operatorname{Re}_{\theta}=1410$," J. Fluid Mech., Vol. 187, 1993, pp. 61-98.

41. Munters, W., Meneveau, C., and Meyers, J., "Shifted Periodic Boundary Conditions for Simulations of WallBounded Turbulent Flows," Phys. Fluids, Vol. 28, No. 2, 2016, pp. 025112.

42. Pirozzoli, S., and Bernardini, M., "Turbulence in Supersonic Boundary Layers at Moderate Reynolds Number," J. of Fluid Mech, Vol. 688, 2011, pp. 120-168.

43. Settles, G. S., Fitzpatrick,T., and Bogdonoff, S. M., "Detailed Study of Attached and Separated Compression Corner Flowfields in High Reynolds Number Supersonic Flow," AIAA J., Vol. 17, No. 6, 1979, pp. 579-585.

44. Ganapathisubramani, B., Clemens, N. T., and Dolling, D. S., "Effects of Upstream Boundary Layer on the Unsteadiness of Shock-Induced Separation,” J. Fluid Mech., Vol. 585, 2007, pp. 369-394.

45. Adams, N. A., "Direct Simulation of the Turbulent Boundary Layer Along a Compression Ramp at $\mathrm{M}=3$ and $\mathrm{Re}_{\theta}=1685, " J$. of Fluid Mech., Vol. 420, 2000, pp. 47-83.

46. Selig, M. S., Andreopoulos, J., Muck, K. C., Dussauge, J. P., and Smits, A. J., "Turbulent Structure in a Shock Wave/Turbulent Boundary- Layer Interaction,” AIAA J., Vol. 27, No. 7, 1989, pp. 862-869. 